\title{
Recognition of Motion Blurred Images Using Invariant Moment and Wavelet Coefficient Feature Space
}

\author{
Soumia Sadasivan
}

\begin{abstract}
Water Reflection is considered to be an important part in the analysis of natural image. Water Reflection images are the most attractive areas for artists and in photography. The existing technique uses the image segmentation and hence recognizes the object from its reflection. However, it cannot recognize certain types of objects. Because of the complex distortion generated by water waves, it needs further enhancement. Hence we propose a novel water reflection recognition technique that overcomes such limitations. The proposed method constructs a feature space composed of motion blur invariant moments in low frequency wavelet space and of wavelet coefficients in high frequency wavelet space. The motion blur in variant moments in low frequency band can distinguish the imperfect symmetry images from other images. The wavelet coefficients in high frequency band can distinguish water reflection images from other imperfect symmetry images. This algorithm can classify water reflection and determine the reflection axis as well as retrieve images with water reflection.
\end{abstract}

Index Terms: Water reflection, imperfect symmetry, motion blur, invariant moments, reflection axis detection.

\section{Introduction}

Symmetry detection and analysis is a fundamental task in computer vision. Most man-made and biological objects exhibit some extent of symmetry. For instance, consider artificial objects such as airplanes and houses or natural ones such as fish and insects. Thus, symmetry is an ubiquitous phenomenon and a useful cue for visual recognition. This is supported by experimental analysis of perceptual grouping and attention of the human visual system.

The two most common types of symmetries are rotational and reflectional symmetries. An object is said to have rotational symmetry of order $\mathrm{K}$ if it is invariant under rotations of $\frac{2 \pi}{\mathrm{K}} \mathrm{k}, \mathrm{k}=0 \ldots \mathrm{K}-1$ about a point denoted the symmetry center. Whereas an object has reflectional symmetry if it is invariant under a reflection transformation about a line, denoted the reflection axis.

Symmetry of an object is a binary characteristic, i.e., an object is either symmetric, or it is not. However, perfect symmetry is rarely observed in real images. Objects, though inherently symmetrical, usually exhibit some deviations from the ideal. Thus, in the real world there is a continuum in symmetry.

Some of the desired properties of any method used for detecting symmetry are the following:

a. avoid a brute-force search in the image;

b. robustness to disjoint contours, including contours formed out of dots;

c. robustness to minor deviations from perfect symmetry;

d. ability to detect multiple symmetries simultaneously;

e. robust to overlap and occlusion;

f. should not assume simple curves (e.g., no self-intersection, convexity, etc.).

Reflection happens between two different medias. The direction of a wavefront at the interface changes so that the wavefront returns into the medium from which it is originated.

Water reflection plays an important role in natural image analysis. Water reflection itself is an exciting natural landscape that attracts artists and photographers, so images with water reflection should be considered as one important category of natural images. The awareness of the existence of water reflection will greatly influence further analysis of an image, such as image segmentation and object recognition. Water reflection is a special case of imperfect reflection symmetry.

In water reflection images, the complexity of water part makes it impossible to keep the consistency between the object part and reflection part perfectly. There exists no such system to address the classification, recognition and detection of water reflection images.

This paper formulates the water reflection recognition as a special case of imperfect reflection symmetry problem. To address the special characteristics of water wave, we construct a novel feature space that is composed of motion blur invariant moments in low-frequency Wavelet space and of Wavelet coefficients in high-frequency Wavelet space. With the help of moment invariants in low-frequency band, we could distinguish the imperfect symmetry images from other images. Utilizing Wavelet coefficients, water reflection images could 
be distinguished from other imperfect symmetry images. Based on the novel feature space, we propose an efficient algorithm including two sub-algorithms: Low-frequency Reflection Cost Minimization (LRCM) and High-frequency Wavelet Coefficients Discrimination (HCCD). This algorithm is effective and reliable to classify water reflection images from other images and to determine the reflection axis. Moreover, this algorithm has lower computational complexity than exhaust algorithm. Also we compare the results of the proposed method with the existing technique in order to illustrate its accuracy in classification of water reflection images, detection of reflection axis and retrieval of images with water reflection.

\section{Related Work}

Lucchese et al. [2] proposed a graceful method in order to analyze the angular properties of an image in Fourier domain. By calculating the Analytic Fourier Mellin Transform Derrode et al. [3] analyzed the symmetries of real objects. These methods uses the concept that Fourier transform preserves the symmetry of images in the fourier domain. Let $\mathrm{I}(\mathbf{x}), \mathbf{x}=[\mathrm{x} y]^{\mathrm{T}} \in \mathrm{R}^{2}$ denote the scalar image of 2-D pattern. Lucchese proved that if an image having reflection symmetry with respect to the reflection axis $\mathrm{y}=\mathrm{x} \times \tan \alpha$, its Fourier transform I $(\omega), \omega \in R^{2}$ has the same reflection symmetry with respect to the line $\omega_{y}=\omega_{x} \times \tan \alpha$. The result shows that the original part and the reflected part will be almost similar. But as there is always some blur in the water part, this conclusion is not always true.

Loy et al. [4] used Scale Invariant Feature Transform (SIFT) descriptor that determines a set of feature points $\mathrm{p}_{\mathrm{i}}$. SIFT detects distinctive points with good repeatability. The point vector $\mathrm{p}_{\mathrm{i}}=\left(\mathrm{x}_{\mathrm{i}}, \mathrm{y}_{\mathrm{i}}, \phi_{\mathrm{i}}, \mathrm{s}_{\mathrm{i}}\right)$ assigned to (optionally) scale. Scale need only be determined if a scale-invariant feature detection method is used. Orientation, however, must be determined as it is central to the evaluation of symmetry. A feature descriptor $k_{i}$ is generated for each feature point, encoding the local appearance of the feature after its orientation (and scale) has been normalized.

A set of mirrored feature descriptors $\mathrm{m}_{\mathrm{i}}$ is generated. Matches are then sort between the features ki and the mirrored features $m_{j}$ to form a set of $\left(p_{i}, p_{j}\right)$ pairs of potentially symmetric features. The SIFT saliency points are matched in pairs between the object and its reflection. But it is difficult to recognize the water reflection by matching the SIFT points.

Zhang et al. [5] proposed a descriptor called Flip Invariant Shape (FIS) descriptor that handles the ripple effects and other complex geometric distortions in water reflection. The proposed method detects water reflection images from unsegmented natural images. The proposed descriptor avoids the extra computation of a mirror descriptor. The leveraged boundary extraction and TPS model updating algorithms effectively handle the ripple effects and the background clutters which often appear in the natural images. First a method that efficiently suppresses background clutters, extract the maximally stable boundaries. Second sample a fixed number of points from the stable boundary. Then, compute the distribution of samples in the image using a logpolar representation. As the log-polar coordinate system has been divided into 60 bins, this descriptor votes the number of sample points in each bin to obtain the bin with the maximum number (MNB) of sample points and the one with the secondary maximum (SMNB). The rotation direction is indicated by the direction from MNB to SMNB. The $\chi 2$ distance is utilized to compute the similarity of the sample points. Thin plate spline (TPS) model is used to further improve the matching result.

Zhong et al. [1] considered water reflection as a special case of imperfect reflection symmetry. He constructed a novel feature space that is composed of motion blur invariant moments in low-frequency Curvelet space and of Curvelet coefficients in high-frequency Curvelet space. It is possible to distinguish the imperfect symmetry images from other images using moment invariants in low-frequency band. Also with the help of Curvelet coefficients in high-frequency band, water reflection images could be distinguished from other imperfect symmetry images.

Stern et al. [6] proposed two methods for the recognition of motion-blurred images. In the first method assuming that the motion function and direction during the exposure are given, develop the relation between the blurred-image moments and the original-image moments based on the motion function only. The recognition is carried out by comparing the moments of the restored image against the moments of the image database. It is demonstrated that the motion PSF moments are equal to the motion function moments. Based on this relation, the central moments of the original image are restored from the observed image central moments and the motion function. The restored moments are compared against the moments of the images in the database to find the best match. In the second method the motion function is not known. In this case image moments that are invariant with respect to the motion blur are identified, and only these moments are used for recognition. This method is based on the fact that moments in directions perpendicular to linear motion are invariant to motion blur. 


\section{A. Reflectional symmetry}

\section{Proposed System}

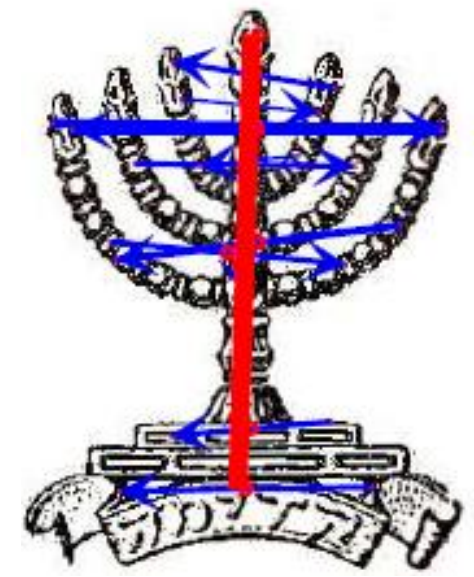

Fig. 1. Reflectional symmetry of order one.

Definition (Reflectional symmetry): A set $\mathrm{S} \in \mathrm{R}^{\mathrm{n}}$ is reflectionally symmetric with respect to the vector (reflection axis) $<\cos \alpha_{0}, \sin \alpha_{0}>$ with a reflectional transform

$T_{D K}$, if for every $x_{i} \in S$, there exist $x_{j} \in S$, such that

where for $x_{i} \in R^{2}, T_{D K}$ is given by

$$
x_{j}=T_{D_{K}} x_{i}
$$

$$
\mathrm{T}_{\mathrm{D}_{\mathrm{K}}}(\mathrm{x}, \mathrm{y})=\left(\begin{array}{cccc}
\cos 2 \alpha_{0} & \sin 2 \alpha_{0} & 0 & \mathrm{x} \\
\sin 2 \alpha_{0} & -\cos 2 \alpha_{0} & 0
\end{array}\right)\left(\begin{array}{l}
\mathrm{y}
\end{array}\right)
$$

A set $\mathrm{S}$ has reflectional symmetry of order $\mathrm{K}$, if there are $\mathrm{K}$ angles $\alpha_{\mathrm{k}}$ that satisfy Eq. 1.

An example of reflectional symmetry is given in Fig.1. where $\alpha_{0}$ is the angle of the reflection axis, and Eq.2 implies that

$$
\operatorname{det}\left(\mathrm{T}_{\mathrm{D}_{\mathrm{K}}}\right)=-1
$$

the points on the symmetry axis form an invariant set $X_{R}$ that corresponds to the eigenspace of

$$
\mathrm{T}_{\mathrm{D}_{\mathrm{K}}} \mathrm{X}_{\mathrm{R}}=\mathrm{X}_{\mathrm{R}}
$$

\section{B. Motion Blur}

Motion blur is the apparent streaking of rapidly moving objects in a still image or a sequence of images such as a movie or animation. It results when the image being recorded changes during the recording of a single frame, either due to rapid movement or long exposure [7]. The formation model for the motion blur is:

$$
\mathrm{g}(\mathrm{x})=\mathrm{I}(\mathrm{x}) * \mathrm{~h}(\mathrm{x})+\mathrm{n}(\mathrm{x})
$$

where $\mathbf{x}=(\mathrm{x}, \mathrm{y}) \in \mathrm{R} 2$ denotes the coordinates of an image pixel, I denotes the original image, $\mathrm{h}(\mathbf{x})$ is the point spread function (psf), $\mathrm{n}(\mathbf{x})$ is additive noise, $\mathrm{g}$ represents the observed image, and the symbol $*$ stands for the 2D convolution operation.

In water reflection, motion blur occurs when there is interplay between the oscillation speed of the surface waves and the camera's limited shutter speed. There is zero motion blur, if image exhibits a perfect instant in time. But this does not happen in the real case. Usually, when a sensor creates an image, that image does not represent a single instant of time. And a fast moving object or a longer exposure time may result in blurring artifacts. Shutter speed or exposure time is the effective length of time a sensor's shutter is open [8]. The human eye can form 10-12 images per second [9]. The agreed standards of cameras for shutter speeds are from $1 / 1000$ second to 1 second [27]. The motion in image is given as

$$
\Delta \mathrm{d}=\Delta \mathrm{x} \times(\mathrm{f} / \mathrm{u})
$$

Where $\Delta \mathrm{x}$ is the motion of object, $\mathrm{f}$ is the focal length and $\mathrm{u}$ is the object distance.

Motion blur changes the image features needed for feature based recognition techniques [30]. This results in some loss of information and energy in high-frequency band. The change of high-frequency 
information in water reflection is one reason for the invalidity of existing global algorithms in Fourier domain [15]. Using deconvolution motion blur can be removed. The main idea in deconvolution is to calculate the point spread function, assuming that the velocity and direction of motion blur is unique [32], [33].

\section{Moment Invariant}

The problem of recognition of objects and patterns that are deformed in various ways can be solved using invariant features. Its basic idea is to describe the objects by a set of measurable quantities called invariants that are insensitive to particular deformations and that provide enough discrimination power to distinguish objects belonging to different classes. From a mathematical point of view, invariant $I$ is a functional defined on the space of all admissible image functions that does not change its value under degradation operator $D$, i.e. that satisfies the condition I (f ) $=I(D(f))$ for any image function $f$. This property is called invariance. In short I (f) should be significantly same as I (D(f)). Another desirable property of I, as important as invariance, is discriminability. For objects belonging to different classes, I must have significantly different values. Clearly, these two requirements are antagonistic the broader the invariance, the less discrimination power and vice versa. Choosing a proper tradeoff between invariance and discrimination power is a very important task in feature based object recognition.

Usually, one invariant does not provide enough discrimination power and several invariants $\mathrm{I}_{1}, . ., \mathrm{I}_{\mathrm{n}}$ must be used simultaneously. Then, we speak about an invariant vector. In this way, each object is represented by a point in an n-dimensional metric space called feature space or invariant space.

Moment invariants are special functions of image moments. Moment invariants have become one of the most important and most frequently used shape descriptors. Even though they suffer from certain intrinsic limitations (the worst of which is their globalness, which prevents direct utilization for occluded object recognition), they frequently serve as "first-choice descriptors" and as a reference method for evaluating the performance of other shape descriptors.

Image moments are weighted averages (moments) of the image pixels' intensities, or functions of those moments, usually chosen to have some attractive property or interpretation [35]. Compared with color histogram, the shift of moment due to the change of illumination will be minimal [36], which also often happens in water part. Moments are scalar quantities used to characterize a function and to capture its significant features.

The first problem is how to distinguish imperfect symmetry images from other images. The proposed feature space has its first component as motion blur invariant moments in low-frequency Wavelet space. This low-frequency channel is used to distinguish imperfect symmetry images from other images. In moment invariants feature space, the difference between the object part and the distorted part due to motion blur is not large.

Definition: General moment $M^{(f)}{ }_{p q}$ of an image $I(x, y)$, where $p$, $q$ are non-negative integers and $r=p+q$ is called the order of the moment, defined as

$$
\mathrm{M}_{\mathrm{pq}}^{(\mathrm{f})}=\iint_{\mathrm{D}} \mathrm{p}_{\mathrm{pq}}(\mathrm{x}, \mathrm{y}) \mathrm{I}(\mathrm{x}, \mathrm{y}) \mathrm{dxdy}
$$

where $\mathrm{p}_{00}(\mathrm{x}, \mathrm{y}), \mathrm{p}_{10}(\mathrm{x}, \mathrm{y}), \ldots, \mathrm{p}_{\mathrm{kj}}(\mathrm{x}, \mathrm{y}), \ldots$ are polynomial basis functions defined on $\mathrm{D}$.

Depending on the polynomial basis used, we recognize various systems of moments.

The most common choice is a standard power basis $p_{k j}(x, y)=x^{k} y^{j}$ that leads to geometric moment:

$$
\mathrm{m}_{\mathrm{pq}}=\iint_{-\infty}^{\infty} \mathrm{x}^{\mathrm{p}} \mathrm{y}^{\mathrm{q}} \mathrm{I}(\mathrm{x}, \mathrm{y}) \mathrm{dxdy}
$$

Geometric moments of low orders have an intuitive meaning $-\mathrm{m}_{00}$ is a "mass" of the image (for binary images, $\mathrm{m}_{00}$ is an area of the object), $\mathrm{m}_{10} / \mathrm{m}_{00}$ and $\mathrm{m}_{01} / \mathrm{m}_{00}$ define the center of gravity or centroid of the image. Second-order moments $\mathrm{m} 20$ and $\mathrm{m} 02$ describe the "distribution of mass" of the image with respect to the coordinate axes. In mechanics they are called the moments of inertia. Another popular mechanical quantity, the

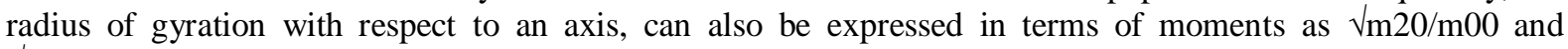

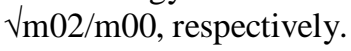

If the image is considered a probability density function (pdf) (i.e. its values are normalized such that $\mathrm{m}_{00}=1$ ), then $\mathrm{m}_{10}$ and $\mathrm{m}_{01}$ are the mean values. In case of zero means, $\mathrm{m}_{20}$ and $\mathrm{m}_{02}$ are variances of horizontal and vertical projections and $\mathrm{m}_{11}$ is a covariance between them. In this way, the second-order moments define the orientation of the image. Second-order geometric moments can be used to find the normalized position of an image. 
The central moments are defined as:

$$
\mu_{\mathrm{pq}}=\int_{-\infty}^{\infty}\left(x-x^{\prime}\right)^{\mathrm{p}}\left(y-y^{\prime}\right)^{\mathrm{q}} \mathrm{I}(\mathrm{x}, \mathrm{y}) \mathrm{dxdy}
$$

where $x^{\prime}=m_{10} / m_{00}$ and $y^{\prime}=m_{01} / m_{00}$ are the components of the centroid. If I is a digital image, Eq. 8 and Eq. 9 are changed to Eq. 10 and Eq. 11.

$$
\begin{gathered}
\mathrm{m}_{\mathrm{pq}}=\sum_{\mathrm{x}} \sum_{\mathrm{y}} \mathrm{x}^{\mathrm{p}} \mathrm{y}^{\mathrm{q}} \mathrm{I}(\mathrm{x}, \mathrm{y}) \\
\mu_{\mathrm{pq}}=\sum_{\mathrm{x}} \sum_{\mathrm{y}}\left(\mathrm{x}-\mathrm{x}^{\prime}\right)^{\mathrm{p}}\left(\mathrm{y}-\mathrm{y}^{\prime}\right)^{\mathrm{q}} \mathrm{I}(\mathrm{x}, \mathrm{y})
\end{gathered}
$$

Moments $\eta_{\mathrm{pq}}$ where $\mathrm{p}+\mathrm{q} \geq 2$ can be constructed to be invariant to both translation and changes in scale by dividing the corresponding central moment by the properly scaled $(00)^{\text {th }}$ moment, using the following formula:

$$
\eta_{\mathrm{pq}}=\frac{\mu_{\mathrm{pq}}}{\mu_{00}^{\left(1+\frac{\mathrm{p}+\mathrm{q}}{2}\right)}}
$$

Based on [37], the following four moment invariants could be proved invariant to linear motion convolution. Therefore, these moment invariants are also invariant to motion blur.

$I_{\mathrm{m} 1}=\left(\eta_{30}-3 \eta_{12}\right)^{2}+\left(3 \eta_{21}-\eta_{03}\right)^{2}$

$\mathrm{IR}_{\mathrm{m} 2}=\left(\eta_{30}+\eta_{12}\right)^{2}+\left(\eta_{21}+\eta_{03}\right)^{2}$

$\operatorname{IR}_{\mathrm{m} 3}=\left(\eta_{30}-3 \eta_{12}\right)\left(\eta_{30}+\eta_{12}\right)\left[\left(\eta_{30}+\eta_{12}\right)^{2}-3\left(\eta_{21}+\eta_{03}\right)^{2}\right]+\left(3 \eta_{21}-\eta_{03}\right)\left(\eta_{21}+\eta_{03}\right)\left[3\left(\eta_{30}+\eta_{12}\right)^{2}-\right.$ $\left.\left(\eta_{21}+\eta_{03}\right)^{2}\right]$

$\mathrm{IR}_{\mathrm{m} 4}=\left(3 \eta_{21}-\eta_{03}\right)\left(\eta_{30}+\eta_{12}\right)\left[\left(\eta_{30}+\eta_{12}\right)^{2}-3\left(\eta_{21}+\eta_{03}\right)^{2}\right]+\left(\eta_{30}-3 \eta_{12}\right)\left(\eta_{21}+\eta_{03}\right)\left[3\left(\eta_{30}+\eta_{12}\right)^{2}-\right.$ $\left.\left(\eta_{21}+\eta_{03}\right)^{2}\right]$

\section{Wavelet Transformation}

The second problem is how to distinguish water reflection images from other imperfect symmetry images. For this end, we utilize the Wavelet coefficients in high-frequency as one component of proposed feature descriptors. The wavelet transform (WT) has gained widespread acceptance in signal processing and image compression. Because of their inherent multi-resolution nature, wavelet-coding schemes are especially suitable for applications where scalability and tolerable degradation are important. Wavelet transform decomposes a signal into a set of basis functions. These basis functions are called wavelets. Wavelets are obtained from a single prototype wavelet $\mathrm{y}(\mathrm{t})$ called mother wavelet by dilations and shifting:

$$
\psi_{\mathrm{a} . \mathrm{b}}(\mathrm{t})=\frac{1}{\sqrt{\mathrm{a}}} \psi \frac{(\mathrm{t}-\mathrm{b})}{\mathrm{a}}
$$

where $\mathrm{a}$ is the scaling parameter and $\mathrm{b}$ is the shifting parameter.

The wavelet transform is computed separately for different segments of the time-domain signal at different frequencies. Multi-resolution analysis analyzes the signal at different frequencies giving different resolutions. MRA is designed to give good time resolution and poor frequency resolution at high frequencies and good frequency resolution and poor time resolution at low frequencies. Wavelet transformation is good for signal having high frequency components for short durations and low frequency components for long duration. e.g. images and video frames.

The 1D wavelet transform is given by:

$$
W_{f}(a, b)=\int_{-\infty}^{\infty} x(t) \psi_{a, b}(t) d t
$$

The inverse 1D wavelet transform is given by:

$$
x(t)=\frac{1}{C} \int_{0}^{\infty} \int_{-\infty}^{\infty} W_{f}(a, b) \psi_{a, b}(t) d b \frac{d a}{a^{2}}
$$

where $C=\int_{-\infty}^{\infty} \frac{\psi(\omega)^{2}}{\omega} \mathrm{d} \omega<\infty$

Discrete wavelet transforms (DWT), which transforms a discrete time signal to a discrete wavelet representation. It converts an input series $\mathrm{x}_{0}, \mathrm{x}_{1}, . . \mathrm{x}_{\mathrm{m}}$, into one high-pass wavelet coefficient series and one lowpass wavelet coefficient series (of length $\mathrm{n} / 2$ each) given by: 


$$
\begin{aligned}
& \mathrm{H}_{\mathrm{i}}=\sum_{\substack{\mathrm{m}=0 \\
\mathrm{k}-1}}^{\mathrm{k}-1} \mathrm{x}_{2 \mathrm{i}-\mathrm{m}} \cdot \mathrm{s}_{\mathrm{m}}(\mathrm{z}) \\
& \mathrm{L}_{\mathrm{i}}=\sum_{\mathrm{m}=0}^{\mathrm{x}_{2 \mathrm{i}-\mathrm{m}} \cdot \mathrm{t}_{\mathrm{m}}(\mathrm{z})}
\end{aligned}
$$

where $s_{m}(z)$ and $t_{m}(z)$ are called wavelet filters, $K$ is the length of the filter, and $i=0, \ldots,[n / 2]-1$. In practice, such transformation will be applied recursively on the low-pass series until the desired number of iterations is reached.

The DWT for 2D is performed firstly for all image rows and then for all columns as shown in Fig. 2. Schematic diagram of 2D wavelet transform is given in Fig. 3.

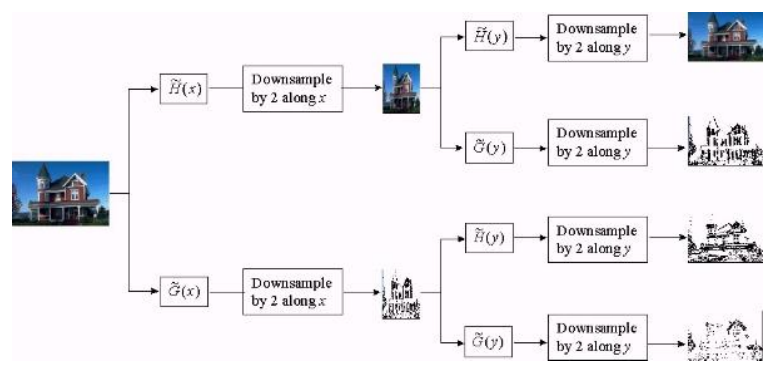

Fig.2.Wavelet decomposition for two dimensional pictures.

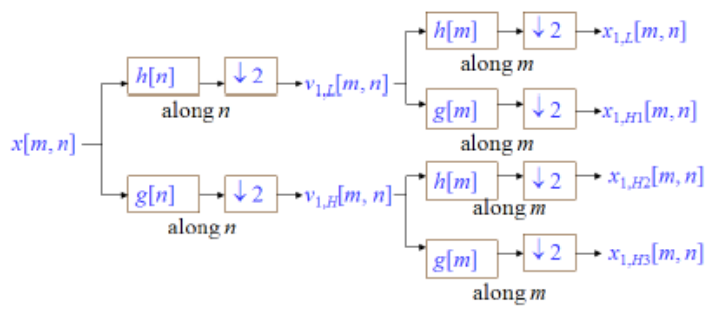

Fig. 3. Schematic diagram for $2 \mathrm{D}$ wavelet transform

The main feature of DWT is multiscale representation of function. By using the wavelets, given function can be analyzed at various levels of resolution. The DWT is also invertible and can be orthogonal. To compute the wavelet features in the first step Harr wavelet is calculated for whole image. As a result of this transform there are 4 sub band images at each scale as illustrated in Fig. 4. This is the general form of 2D wavelet transform. If the scaling and wavelet functions are separable, the summation can be decomposed into two stages. First step is along the $\mathrm{x}$-axis and then calculate along the $\mathrm{y}$-axis. For each axis, we can apply fast wavelet transform to accelerate the speed. A schematic diagram is shown in Fig. 3.4. The two dimensional signal (usually image) is divided into four bands: LL(left-top), HL(right-top), LH(leftbottom) and HH(rightbottom). The HL band indicated the variation along the $\mathrm{x}$-axis while the LH band shows the y-axis variation. Fig. 3.3, 3.4, and 3.5 show the decomposition of a image. The power is more compact in the LL band. In the point of coding, we can spend more bits on the low frequency band and less bit on the high frequency band or even set them to zero.

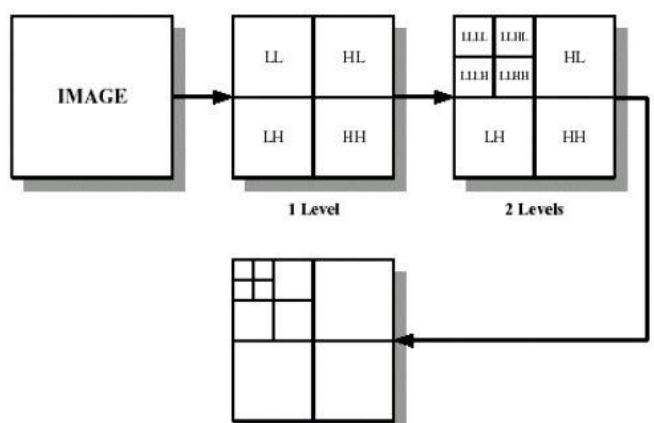

Fig. 3. Sub band images 
The sub-algorithm Low-frequency Reflection Cost Minimization (LRCM) for imperfect symmetry recognition [1] is given.

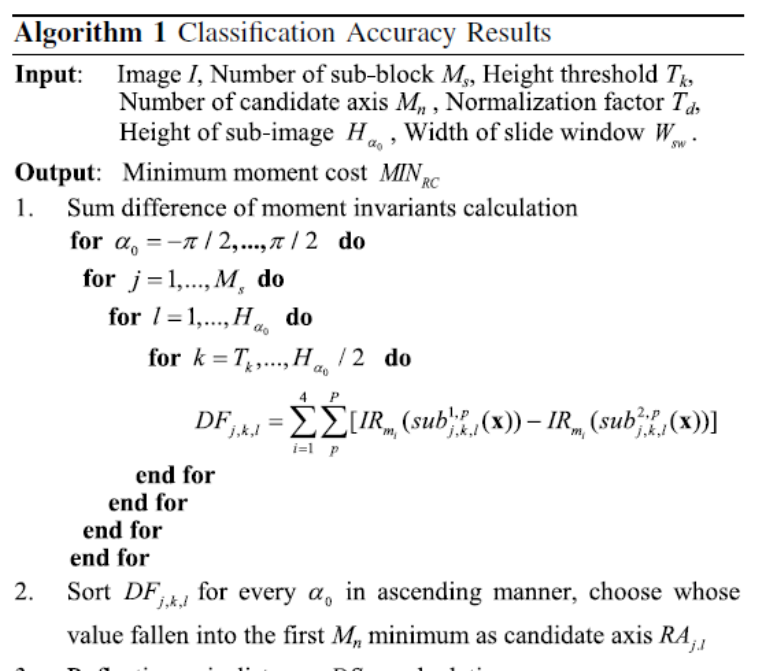

3. Reflection axis distance $D S_{j, l}$ calculation

$$
D S_{j, l}= \begin{cases}\left\|R A_{j, l}-R A_{j+1.1}\right\| / T_{d}+1 & j \leq M_{s}-1 \\ 1 & j=M_{s}\end{cases}
$$

4. Minimum moment cost in stage $K$ calculation

$v_{K}=\min \left[D F_{j, k, l} \times D S_{j, l}\right]$

$$
\text { s.t. } K=j, T_{k} \leq k \leq \frac{H_{a_{0}}}{2}, l \in S W_{m}, 1 \leq m \leq H_{\alpha_{0}}-W_{s v},-\frac{\pi}{2} \leq \alpha_{0} \leq \frac{\pi}{2}
$$

5. Minimum moment cost calculation for every $\alpha_{0}$

$$
\begin{cases}f_{K}\left(\lambda_{K}\right)=\min \left\{v_{K}\left(\lambda_{K}, \mu_{K}\right)+f_{K-1}\left(\lambda_{K-1}\right)\right\} \\ \lambda_{K} \in \Lambda_{K} & K=j, 1 \leq j \leq M_{s} \\ \lambda_{K+1}=\mu_{K} & \end{cases}
$$

6. Minimum moment cost $M I N_{R C}$ calculation

$$
\operatorname{MIN}_{R C}=\min \left(f_{M_{s}}\left(\lambda_{M_{s}}\right)\right), 1 \leq m \leq H_{\alpha_{0}}-W_{s w},-\frac{\pi}{2} \leq \alpha_{0} \leq \frac{\pi}{2}
$$

The flow chart of the proposed method is shown in Fig. 5.

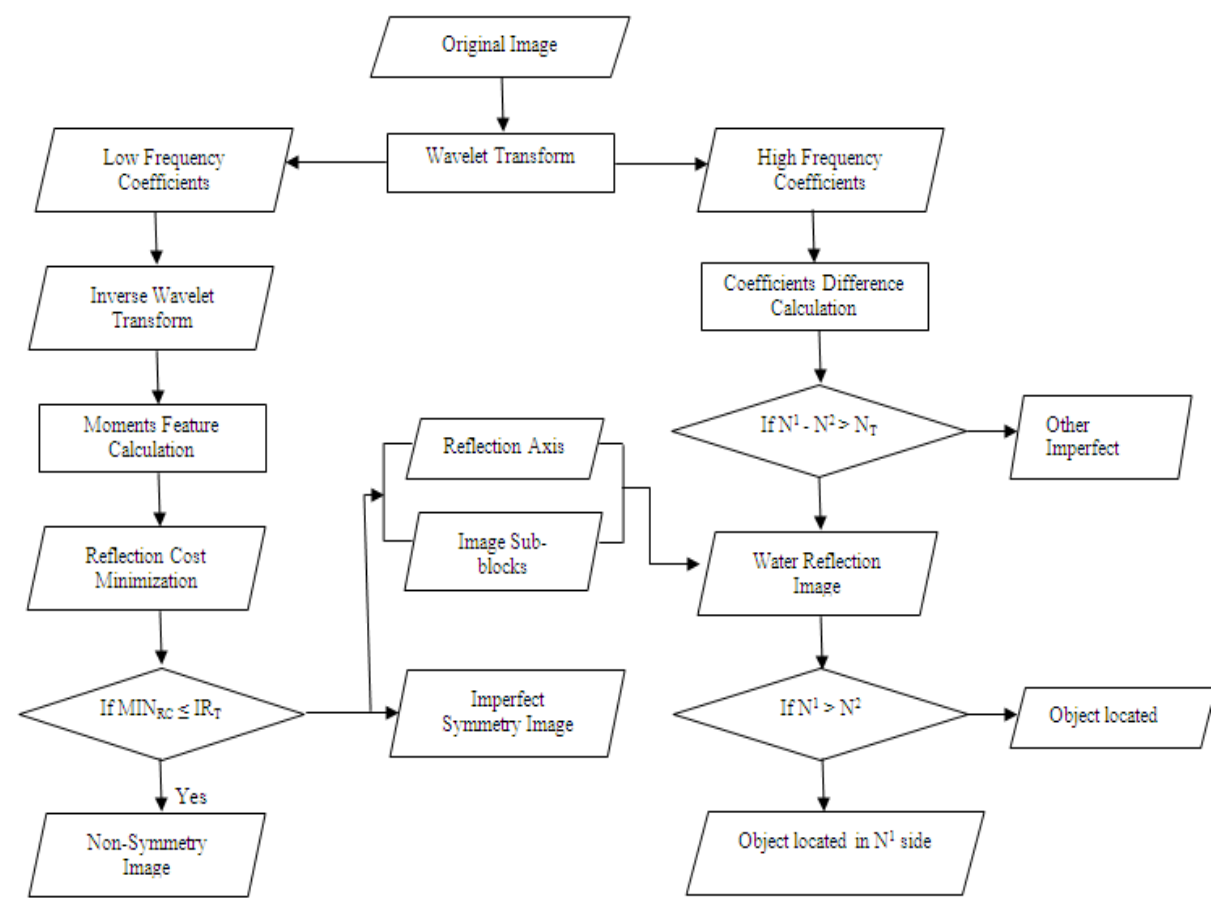

Fig. 5. Flowchart of the proposed method 


\section{Results}

To demonstrate the performance of our proposed technique, we conduct three experiments, including the classification of nature scene images with and without water reflection, the detection of reflection axis, and the retrieval of water reflection images.

\section{A. Water Reflection Image Classification}

In the first experiment, to evaluate the classification accuracy of the proposed technique with respect to the existing technique, we construct a dataset including 20 images with water reflection and 20 nature scene images without water reflection. We subdivide this dataset equally into five folders, and conduct fivefold cross validations for the learning algorithms. Every time, we utilize one folder for testing, and the other four folders for training. If $\mathrm{MIN}_{\mathrm{RC}}$ is below the threshold $\mathrm{IR}_{\mathrm{thresh}}$ which is learnt by binary SVM classifier based on the training dataset, this image is classified as the water reflection image. The classification accuracy results are provided in Fig. 6.

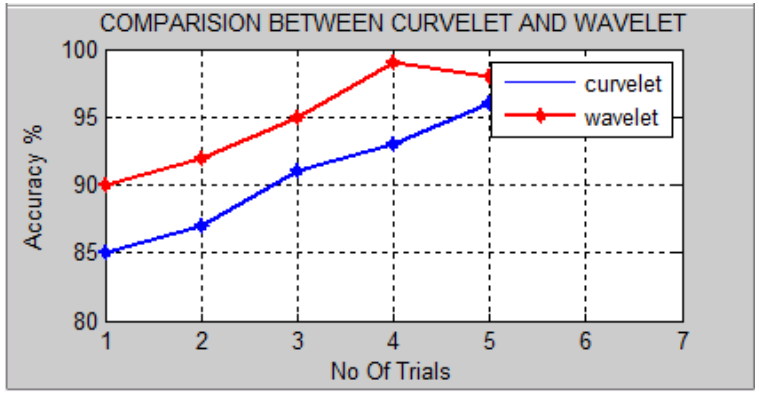

\section{B. Detection the Reflection Axis}

Fig. 6. Classification accuracy

To compare with existing symmetry techniques, the detection experiment is carried out on 20 images with water reflection where reflection axes are provided by human subjects. The goal of this experiment is to detect the reflection axis. One example of detection of reflection axis in water reflection image is shown in Fig. 7.

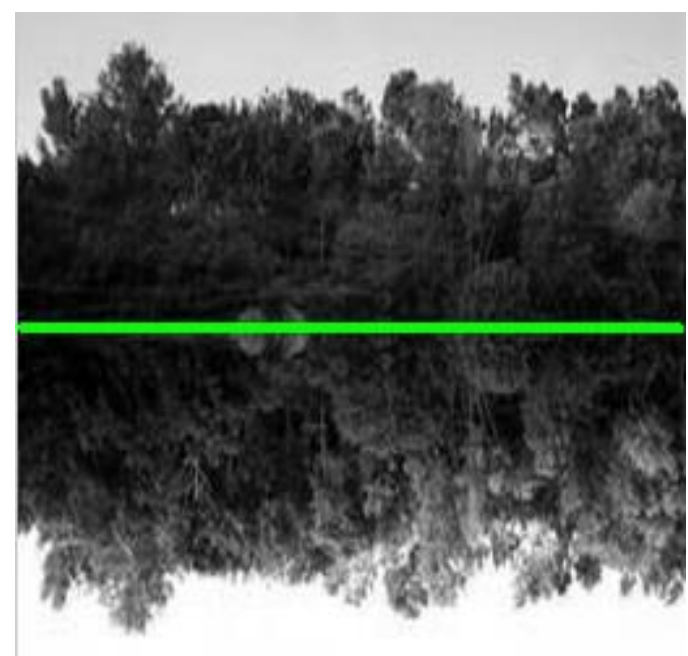

Fig. 7. Axis Image

\section{Conclusion}

Water reflection is a special case of imperfect reflection symmetry problem, but no existing techniques have been proposed to address the task of water reflection image classification. To address this problem, we propose a novel feature space Invariant Moment \& Wavelet Coefficient according to the characteristics of motion blur in water reflection images. An effective and efficient water reflection classification and reflection axis detection technique is then constructed. Experiments and evaluation all confirmed the effectiveness and robustness of our technique, which is more reliable and successful compared with existing feature space and algorithms. 


\section{References}

[1]. Sheng-Hua Zhong, Yan Liu, Yang Liu, and Chang-Sheng Li, "Water Reflection Recognition Based on Motion Blur Invariant Moments ," IEEE Transaction On Image Processing, vol. 22, no. 11, pp. 4301-4313, Feb. 2004.

[2]. L. Lucchese, "Frequency domain classification of cyclic and dihedral symmetries of finite 2-D patterns," Pattern Recognit., vol. 37, no. 12, pp. 2263-2280, Dec. 2004.

[3]. S. Derrode and F. Ghorbel, "Shape analysis and symmetry detection in gray-level objects using the analytical Fourier-Mellin representation," Signal Process., vol. 84, no. 1, pp. 25-39, Jan. 2004.

[4]. G. Loy and J. Eklundh, "Detecting symmetry and symmetric constellations of features," in Proc. Eur. Conf. Comput. Vis., May 2006, pp. 508-521.

[5]. H. Zhang, X. J. Guo, and X. C. Cao, "Water reflection detection using a flip invariant shape detector," in Proc. Int. Conf. Pattern Recognit., Aug. 2010, pp. 633-636.

[6]. A. Stern, I. Kruchakov, E. Yoavi, and N. S. Kopeika, "Recognition of motion-blurred images by use of the method of moments," Appl. Opt., vol. 41, no. 11, pp. 2164-2171, 2002.

[7]. J. Flusser, T. Suk, and S. Saic, "Recognition of images degraded by linear motion blur without restoration," in Computing Supplementum, vol. 11. New York, NY, USA: Springer-Verlag, 1996, pp. 37-51.

[8]. F. R. Sidney, "Camera features," in Manual of Photography: A Textbook of Photographic and Digital Imaging. Waltham, MA, USA: Focal Press, 2000, pp. 131-132.

[9]. P. Read and M. P. Meyer, "Restoration of Motion picture film," in Conservation and Museology. Oxford, U.K.: ButterworthHeinemann, 2000, pp. 24-26. 